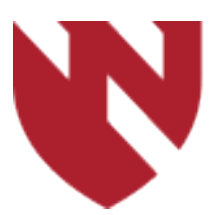

October 2021

\title{
Varicella-Zoster Virus-associated Longitudinally Extensive Transverse Myelitis in an Immunocompetent Adult: An Unusual and Rare Complication of Herpes Zoster
}

\author{
Daniel Crespo \\ University of Nebraska Medical Center \\ Amrita-Amanda Vuppala \\ University of Nebraska Medical Center \\ David Semerad \\ Omaha VA Medical Center \\ John M. Bertoni \\ University of Nebraska Medical Center
}

Tell us how you used this information in this short survey.

Follow this and additional works at: https://digitalcommons.unmc.edu/gmerj

Part of the Higher Education Commons, and the Medicine and Health Sciences Commons

\section{Recommended Citation}

Crespo, D., Vuppala, A., Semerad, D., , Bertoni, J. M. Varicella-Zoster Virus-associated Longitudinally Extensive Transverse Myelitis in an Immunocompetent Adult: An Unusual and Rare Complication of Herpes Zoster. Graduate Medical Education Research Journal. 2021 Oct 04; 3(1). https://digitalcommons.unmc.edu/gmerj/vol3/iss1/36

This Conference Proceeding is brought to you for free and open access by DigitalCommons@UNMC. It has been accepted for inclusion in Graduate Medical Education Research Journal by an authorized editor of DigitalCommons@UNMC.For more information, please contact digitalcommons@unmc.edu. 


\section{Varicella-Zoster Virus-associated Longitudinally Extensive Transverse Myelitis in an Immunocompetent Adult: An Unusual and Rare Complication of Herpes Zoster}

Creative Commons License

(c) (i) (3)

This work is licensed under a Creative Commons Attribution-Noncommercial-No Derivative Works 4.0 License. 
proximal medial tibial plateau were present. He had positive Thessaly and non-painful crepitus with McMurray maneuvers. He was unable to complete a squat or full AROM due to pain. MRI knee revealed acute nondisplaced proximal tibial metaphysis fracturelikely insufficiency fracture, joint effusion, and DJD.

Patient was made NWB and placed in hinged knee brace locked in full extension for 3 weeks and then progressive WB and ROM as typical for tibial plateau fracture management. Calcium \&Vit D supplementation was prescribed, and the patient was referred to endocrinology for bone health optimization. Patient consent was obtained in order to use this information for education purposes.

Conclusion: Bariatric surgery has known risk of negatively affecting bone health. Different types of procedures have expressed variable

\section{Unilateral Livedoid Hyperpigmentation of the Lower Extremity Dillon Clarey ${ }^{1}$, Devor O'Connor ${ }^{2}$, Ashley Wysong ${ }^{1}$ \\ ${ }^{1}$ University of Nebraska Medical Center, College of Medicine, Department of Dermatology ${ }^{2}$ University of Nebraska Medical Center, College of Medicine}

\section{Mentor: Ashley Wysong}

Program: Dermatology

Type: Case Report

Background: Erythema ab igne is a clinical diagnosis that comes with a broad differential diagnosis. Thorough history taking and ruling out of other reticulate dermatoses enables for its diagnosis. It is most often seen in cases of prolonged exposure to heat (space heater, laptop usage, heating blankets).

Case: A 40-year-old female with past history of livedo reticularis, hypertension, type II diabetes, obesity, and traumatic brain injury presented to the emergency department with a 1-month history of left lower extremity rash, edema, and bullae. She denied any remote history of intravenous drug use or chronic heat exposure. Recent outpatient workup for livedo reticularis revealed no evidence of venous/arterial insufficiency or deep venous thrombosis.
Examination demonstrated net-like violaceous patches consistent with livedo reticularis, some non-blanchable, over the left lower extremity. Overlying, there were several tense bullae with clear fluid and other deroofed bullae as well as xerotic scaly plaques. Laboratory workup revealed an elevated erythrocyte sedimentation rate (ESR) and $\mathrm{C}$-reactive protein (CRP) with unremarkable complete blood count (CBC), complete metabolic panel (CMP), antinuclear antibody (ANA), treponema pallidum antibody, and bacterial culture swab. With no immediate need for hospital admission, punch biopsy for hematoxylin and eosin (H\&E) and tissue culture was obtained and triamcinolone $0.1 \%$ cream was started. The patient was discharged home. Biopsy revealed spongiotic dermatitis with prominent papillary dermal edema and no evidence of vasculitis, vasculopathy, or infectious etiology.

At a follow up visit, the patient noted that her home furnace had broken in March of levels of risk and other factors including gender and vitamin regimens can affect long term sequela. Compliance and follow up could have potentially prevented this patient's fracture and overall morbidity, reinforcing their importance.

https://doi.org/10.32873/unmc.dc.gmerj.3.1.025

2020, at which time she purchased a large space heater which she had been using in very close proximity to her left leg. A diagnosis of bullous erythema ab igne with superficial ulcerations was made. Management options discussed included 5-fluorouracil, topical retinoids, and lasers, all of which were declined by the patient. She was instructed to place the space heater at a distance from her left leg. Vaseline, Telfa, Kerlix, and Coban were placed over the affected leg to use until reepithelialization had taken place. Patient consent was obtained for both photography and sharing of history in this case.

Conclusion: This case highlights the broad differential and workup for livedo reticularis, and it emphasizes the importance of thorough history taking and comprehensive physical examination.

https://doi.org/10.32873/unmc.dc.gmerj.3.1.030

\section{Varicella-Zoster Virus-associated Longitudinally Extensive Transverse Myelitis in an Immunocompetent Adult: An Unusual and Rare Complication of Herpes Zoster Daniel Crespo ${ }^{1}$, Amrita-Amanda Vuppala ${ }^{1,2}$, David Semerad ${ }^{3}$, John Bertoni1,4 \\ ${ }^{1}$ University of Nebraska Medical Center, College of Medicine, Department of Neurology \\ ${ }^{2}$ University of Nebraska Medical Center, Truhlsen Eye Institute \\ ${ }^{3}$ Omaha VA Medical Center, Department of Radiology \\ ${ }^{4}$ University of Nebraska Medical Center, College of Medicine, Department of Neurology, Division of Movement Disorders}

Mentor: Amrita-Amanda Vuppala

Program: Neurology

Type: Case Report

Background: Herpes Zoster (HZ) occurs from reactivation of Varicella Zoster Virus (VZV) in dorsal root ganglia. Common neurological complications include cranial neuropathies and encephalitis. Longitudinally extensive transverse myelitis (LETM) has rarely been described in immunocompetent patients. We report a case of VZV-associatedLETM occurring despite a course of acyclovir for HZ.

Case: A 58-year-old immunocompetent male presented with $\mathrm{HZ}$ infection in right $\mathrm{T} 4$ dermatome. He received a course of acyclovir. Three weeks later, he developed right chest numbness attributed to post-herpetic neuralgia and received analgesics. A few days later he presented to ED with bilateral lower extremity weakness and numbness from T4 level. The initial MRI was normal. He again received acyclovir for VZV-associated myelitis despite negative imaging. CSF showed lymphocytic pleocytosis, high VZV IgG levels. Bloodwork/CSF analysis ruled out other infectious/autoimmune etiologies. Five days later, MRI was again unremarkable. He developed rapidly progressive paraplegia. Thirteen days after admission, a third MRI showed a longitudinally extensive transverse myelitis, contrast enhancing and centrally located from C7 to T4. Decreasing the echo time (TE) on the STIR sequence helped make the diagnosis. Plasma exchange was 
attempted but he had an anaphylactic reaction. He was ultimately treated with IV steroids and IVIG with poor recovery. Consent was obtained.

Conclusions: VZV-associated LETM is an unusual complication of treated HZ. Although there is no standard treatment, acyclovir, IVIG and plasma exchange are used as potential treatments, ultimately with poor prognosis. Negative imaging long after symptom onset emphasizes the importance of a good neurologic exam and repeat imaging for diagnosis.

https://doi.org/10.32873/unmc.dc.gmerj.3.1.020

\section{Parkinsonism-Hyperpyrexia Syndrome Secondary to Deep Brain Stimulation Withdrawal Treated with High Doses of Levodopa-Carbidopa: a Rare Life-Threatening Emergency} Daniel Crespo ${ }^{1}$, Kiel Woodward ${ }^{1}$, Miguel Situ-Kcomt ${ }^{1}$

${ }^{1}$ University of Nebraska Medical Center, College of Medicine, Department of Neurological Sciences

Mentor: Miguel Situ-Kcomt

Program: Neurology

Type: Case Report

Background: Parkinsonism-HyperpyrexiaSyndrome (PHS) is an emergency mimicking Neuroleptic-Malignant-Syndrome (NMS) and presents with rigidity, hyperpyrexia, increased creatine phosphokinase (CPK), altered consciousness, autonomic instability. Abrupt discontinuation of dopaminergic therapy in a patient with Parkinson's disease (PD) is the main mechanism causing PHS. This has been generally reported with the sudden interruption of enteral administration of medications but has been anecdotally reported in deep brain stimulation (DBS) withdrawal. Pathophysiology of PHS triggered by DBS discontinuation is poorly understood.

Case: This 56-year-old male had a past medical history of 20 years of Parkinson's disease (PD) status post bilateral STN-DBS 12 years prior and baseline asymmetric tremor, motor fluctuations with dyskinesias, hallucinations, gait imbalance with a Unified PD Rating Scale (UPDRS) of 61. His antiparkinsonian medication regimen consisted of carbidopa-levodopa, ropinirole, trihexyphenidyl, and rivastigmine. He underwent removal of his Implantable Pulse Generator (IPG) due to soft tissue infection. He then presented severe worsening of parkinsonism with a UPDRS of 96. Neurological exam showed severe dysarthria, persistent head rotational tremor, severe tremor at the bilateral elbow and wrist joints, and moderate tremor at the bilateral ankles. Severe cogwheeling rigidity was present in all extremities.

Although already on adequate directed antibiotics for the infection, he continued to have fevers (102.2 F), hypertension (223 mmHg of systolic blood pressure), tachycardia (heart rate of 150's), and a CPK above 5000. He developed severe dysphagia which prevented him from swallowing medications. After placement of a Nasogastric tube, levodopa-carbidopa dose was restarted and tripled. His tremors slowly improved, dysautonomia resolved, CPK levels normalized.

Conclusions: PHS secondary to DBSwithdrawal is a rare and life-threatening condition occurring upon abrupt DBS cessation. We present a case of PHS in the setting of DBS-withdrawal secondary to IPG infection and removal that resolved with aggressive dopaminergic medication regimen without re-implantation of IPG. Ultimately, DBS restoration is the definitive treatment.

https://doi.org/10.32873/unmc.dc.gmerj.3.1.031

\section{Bilateral Limb-Shaking Transient Ischemic Attack Secondary to Bilateral Anterior Cerebral Artery Stenosis and Right A3 Thrombosis \\ Daniel Crespo ${ }^{1}$, Michael Pichler ${ }^{1}$}

${ }^{1}$ University of Nebraska Medical Center, College of Medicine, Department of Neurological Sciences

Mentor: Michael Pichler

Program: Neurology

Type: Case Report

Background: Limb-shaking Transient Ischemic Attack (TIA) is a very rare presentation of cerebral arterial occlusion. It presents as involuntary abnormal movements that occur in one or more limbs usually triggered by orthostasis and cerebral hypoperfusion. This has been described in unilateral or bilateral carotid artery occlusions but to the best of our knowledge, this is the first case of Limb-Shaking TIA secondary to bilateral anterior cerebral artery (ACA) occlusions.

Case: This is a 76-year-old male with significant cardiovascular comorbidities who presented with three months of recurrent falls preceded by 30 seconds of weakness and shaking of bilateral legs. He did not have any prodromal symptoms or loss of awareness associated with these episodes that occurred several times per day. After a few minutes of being in the ground, he would regain complete strength and would be able to stand up independently. He was found to have orthostatic hypotension during these episodes. Vascular imaging showed a complex anatomy of the anterior cerebral arteries, with fusiform dilation of the right A2 segment, a thrombosed right A3 segment and stenosed left ACA. MRI of the brain did not show diffusion restriction consistent with ischemic injury. He was also found to have lumbar spinal stenosis which was thought to contribute to the weakness. He was started on dual antiplatelet therapy and high intensity statins. His symptoms responded to hydration and careful ambulation with compression stockings.

Conclusion: Bilateral limb-shaking TIAs are a very unusual presentation of cerebral hypoperfusion. They are often misdiagnosed as focal seizures with preserved awareness or even movement disorders such as myoclonus. It is important to identify triggers such as orthostatic hypotension that can be often reproduced at bedside. Management involves careful blood pressure control, avoidance of precipitating factors and consideration of revascularization if applicable.

https://doi.org/10.32873/unmc.dc.gmerj.3.1.019 